Document downloaded from:

http://hdl.handle.net/10251/178933

This paper must be cited as:

Cebrecos, A.; García-Garrigós, JJ.; Descals, A.; Jimenez, N.; Benlloch Baviera, JM.; Camarena Femenia, F. (2020). Dynamic beamforming for large area scan in array-based photoacoustic microscopy. IEEE. 1-4. https://doi.org/10.1109/IUS46767.2020.9251519

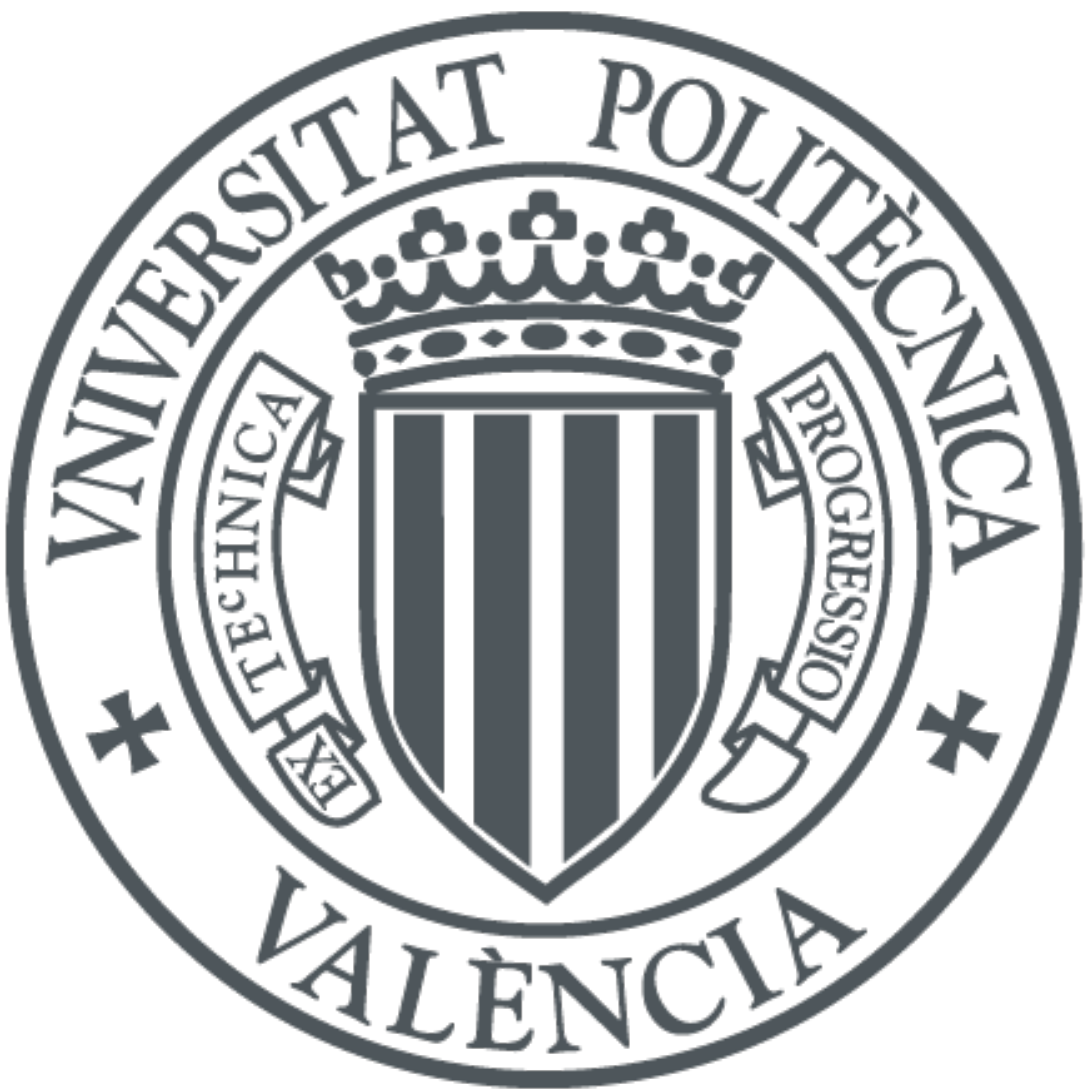

The final publication is available at

https://doi.org/10.1109/IUS46767.2020.9251519

Copyright IEEE

Additional Information 


\title{
Dynamic beamforming for large area scan in array-based photoacoustic microscopy
}

\author{
Alejandro Cebrecos*, Juan J. García-Garrigós, Andreu Descals, \\ Noé Jiménez, José M. Benlloch and Francisco Camarena \\ Instituto de Instrumentación para Imagen Molecular (i3M), \\ Universitat Politècnica de València - CSIC, València, Spain \\ *Email: alcebrui@upv.es
}

\begin{abstract}
We explore the use of a beamforming method intended for large-area scanning in optical-resolution photoacoustic microscopy. It has been evaluated in a experimental setup that comprises a low-cost laser diode and a phase array with a 128elements linear probe. Three different beamforming strategies are discussed: no-beamforming, static beamforming and dynamic beamforming. The method has been tested in gelatine-based phantoms as well as ex-vivo organs. Results show that, compared with the other two, dynamic beamforming increases up to $15 \mathrm{~dB}$ and homogenizes signal-to-noise ratio (SNR) along images of roughly $1 \mathrm{~cm}^{2}$. The method and system presented here could be the baseline for more advanced array-based systems that leverage the low-cost laser sources for clinical applications.
\end{abstract}

Index Terms-photoacoustic imaging, OR-PAM, large area scan, array-based beamforming, pulsed laser diode

\section{INTRODUCTION}

Photoacoustic microscopy (PAM) is a hybrid (opticalacoustical) technique that has been proven capable of generating images of the microvasculature of superficial tissues. The mapping is commonly done by means of raster-scanning, without reconstruction algorithms [1]. PAM is furthered classified into two main modalities depending on imaging depth, which defines different focusing strategies: acoustic resolution (AR-PAM) and optical resolution (OR-PAM). In ARPAM resolution is determined by the ultrasound propagation and detection, while in OR-PAM is the size of the optical spot used to generate the PA waves what determines the lateral resolution, hence the importance of tightly focusing the laser beam. Typically, OR-PAM experimental setups use solid state lasers such as Nd:YAG or Ti:Sapphire [2]. As an alternative light source, pulsed laser diodes (PLD) as well as LED have been an upward trend for the last fifteen years $[3,4]$. While lightweight and inexpensive, the resulting signal usually requires multiple averaging due to the comparatively low energy delivered. Most commonly used US detectors are either focused or planar piezoelectric detectors. All-optical alternatives based on optical fiber have been explored as a good compromise between sensibility and higher field of view (FOV) and acceptance angle [5]. Piezoelectric arrays are occasionally used in PAM for different purposes such as volumetric [6] or real-time imaging [7]. For instance, Hajireza et al. [8] reported a microendoscopy setup that combined a linear array with delay-and-sum beamforming to increase the signal-tonoise ratio (SNR). In this study, we quantify the improvement in SNR using delay-and-sum beamforming in an array-based OR-PAM system and, thus, improving the FOV of the system. An array-based PLD OR-PAM system for large-area scanning is demonstrated. A dynamic beamforming method is used to increase the all around SNR and, as a result, synchronize both optical and acoustical foci. This method allows to scan larger areas without the need of water as a coupling medium, specially when combined with faster scanning devices such as MEMS or galvanometer scanners.

\section{MATERIALS AND METHODS}

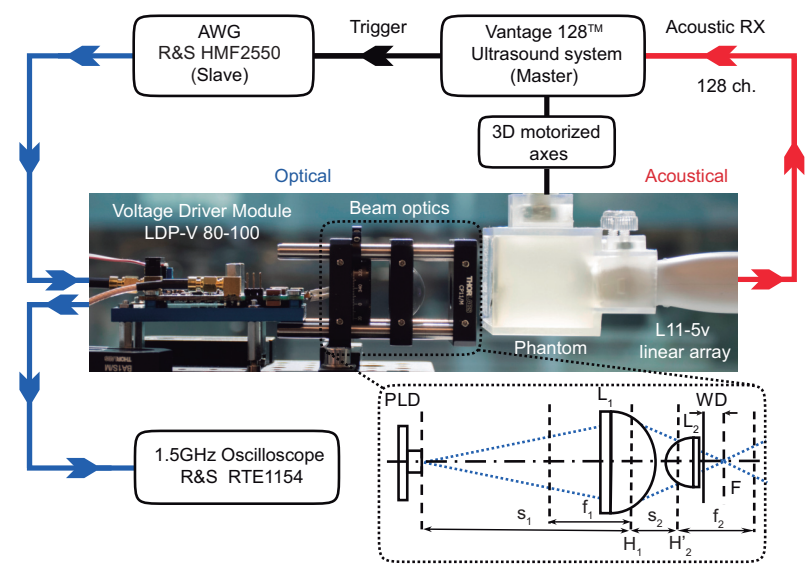

Fig. 1. Main components of the OR-PAM system. Optical part (blue) starts with arbitrary waveform generator, which receives the trigger from the acquisition system and continues with the driver module, pulsed laser diode and associated optics (detailed schema) and oscilloscope. The acoustic part (red) is composed of the ultrasound probe, the Vantage acquisition system, and the 3D stage to raster scan the sample.

The experimental setup, as shown in Fig. 1, is composed of a high-power PLD (905D5S2L3J08X, Laser Components, Germany) of $650 \mathrm{~W}$ and $905 \pm 10 \mathrm{~nm}$ central wavelength coaxially aligned with a 128-element linear US probe (L11$5 \mathrm{v}$, Verasonics, USA). A set of lenses are used to concentrate and focalize the highly divergent source of light into a tight spot delivered to the target, as depicted in 1 . The acquisition system (Vantage $256^{\mathrm{TM}}$, Verasonics USA) obtains the signals corresponding to each channel of the US array and synchronizes the laser excitation with the scanning of the sample by a $3 \mathrm{D}$ stage. 

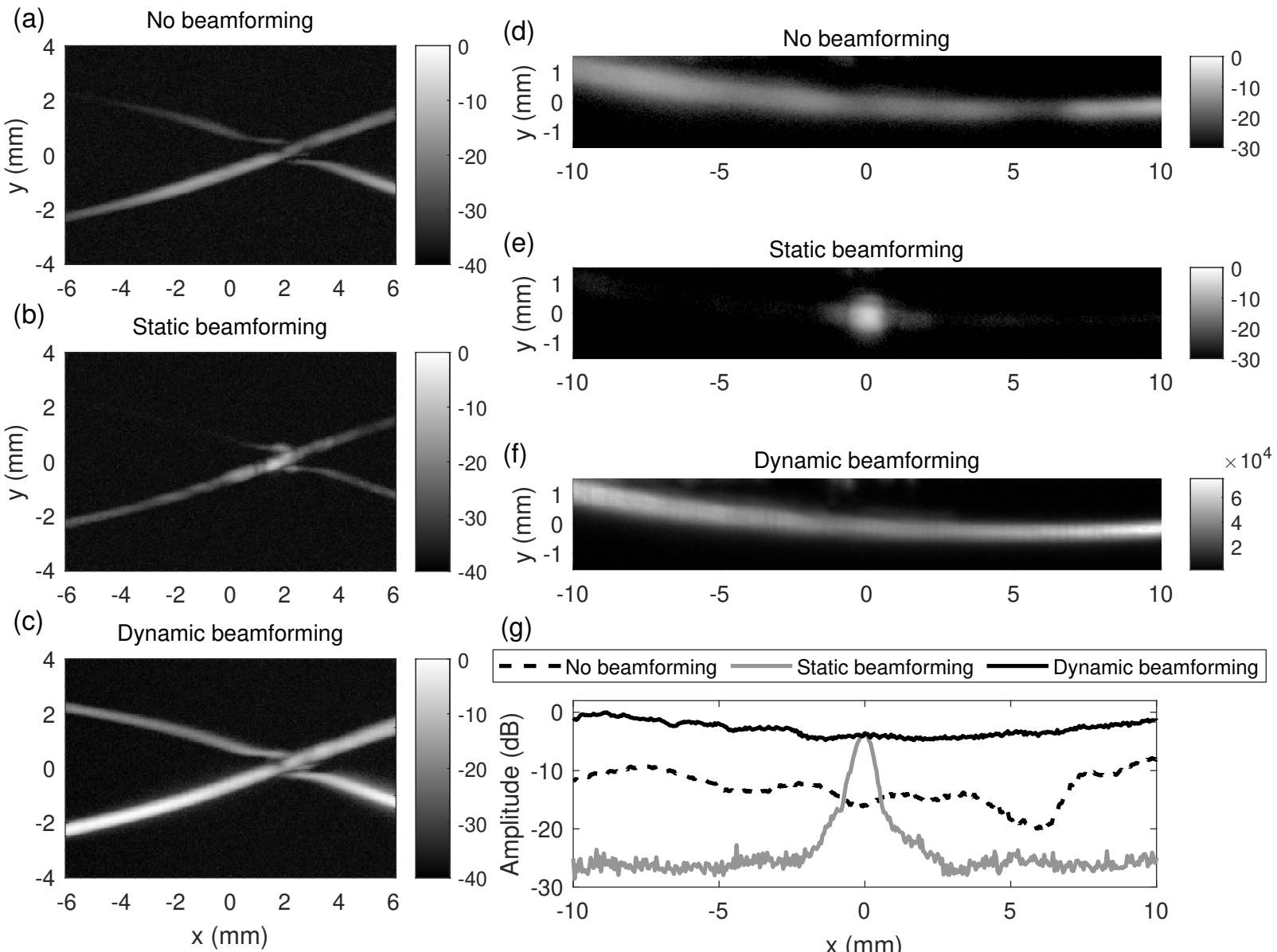

(g)
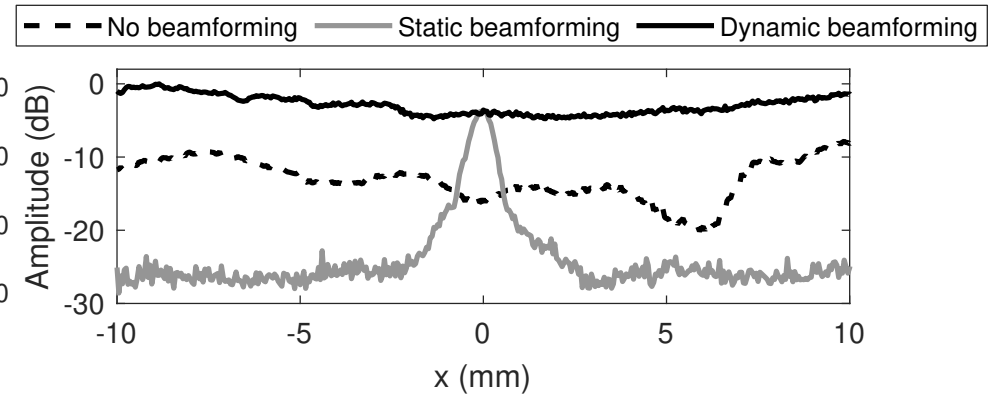

Fig. 2. 2D photoacoustic images from gelatine phantoms with ink tubes. 2D images processed according to three different strategies: (a) no-beamforming, (b) static beamforming and (c) dynamic beamforming. Horizontal tubes scanning to determine SNR along the longitudinal axis of the probe (d), (e) and (f). Comparative plot of the amplitude of the signal along the main axis for each beamforming method $(\mathrm{g})$.

Phantoms are made of gelatine $(6 \% \mathrm{~m} / \mathrm{V})$ and produced in custom-made containers, with polyethylene tubes $(0.42 \mathrm{~mm}$ in-diameter) filled with India ink inserted and spread close to the surface. It should be noted that the US probe is in contact with the gelatine with the only addition of coupling gel for acoustic-impedance matching. Thus, the probe does not move with respect to the phantom and there is no need for water as a coupling medium, as opposed to other OR-PAM implementations [9].

The resulting signals are processed in MATLAB (Mathworks, USA) to generate maximum intensity projection (MIP) representations according to three different strategies: (1) nobeamforming, (2) static beamforming and (3) dynamic beamforming. In the no-beamforming method, for every scanning point, 128 signals (one for every channel of the US probe) are added together as they are received, without considering the time delay. In static beamforming, these same signals are delayed in order to reflect a single-element focused transducer scenario. Finally, dynamic beamforming applies a delay-andsum (DAS) algorithm to the signals registered in each scanning point. This applies a delay based on the distance between the source and each of the 128 channels of the probe [10].

\section{RESULTS}

MIP images of gelatine-phantoms are obtained and processed accordingly to the three described strategies.

To measure the lateral resolution of the system, a highly absorbing $180-\mu \mathrm{m}$ vinyl strip was embedded in the phantom at around $1 \mathrm{~mm}$ beneath its surface and partially imaged. The border of the strip was scanned horizontal and vertically with a $10 \mu \mathrm{m}$ steps. Several measurements were 256-times averaged and combined to obtain the resulting edge spread function (ESF). Thus, the lateral resolution of the system as determined by the full width at half maximum (FWHM) of the adjusted line spread function (LSF) is determined to be $200 \mu \mathrm{m}$ and $119 \mu \mathrm{m}$ along the horizontal and vertical axis, respectively.

2D-scans of gelatine phantoms including polyethylene tubes filled with black India ink are presented in Fig. 2 (a-c). The scanned area was $1.2 \times 0.8 \mathrm{~cm}$. Acquisitions were processed following the three strategies described. The dynamic beamforming method clearly achieves higher SNR and better contrast, while the other two (no-beamforming and static 
beamforming) fail to achieve a uniform contrast all around the area imaged.

Moreover, a single polyethylene tube was scanned to evaluate the SNR variation along the probe longitudinal axis Fig. 2 (d-f). Results show how the dynamic beamforming strategy offers better contrast with less variation (around $3 \mathrm{~dB}$ ) along the main axis. When compared with a no-beamforming strategy, the later shows a 10 to $20 \mathrm{~dB}$ reduction. While static beamforming has the same SNR at the focus, which is expected, it decreases rapidly with distance. Note that some of the divergences in the SNR can be explained by small variation in the positioning of the tube with respect to the $\mathrm{z}$-axis.
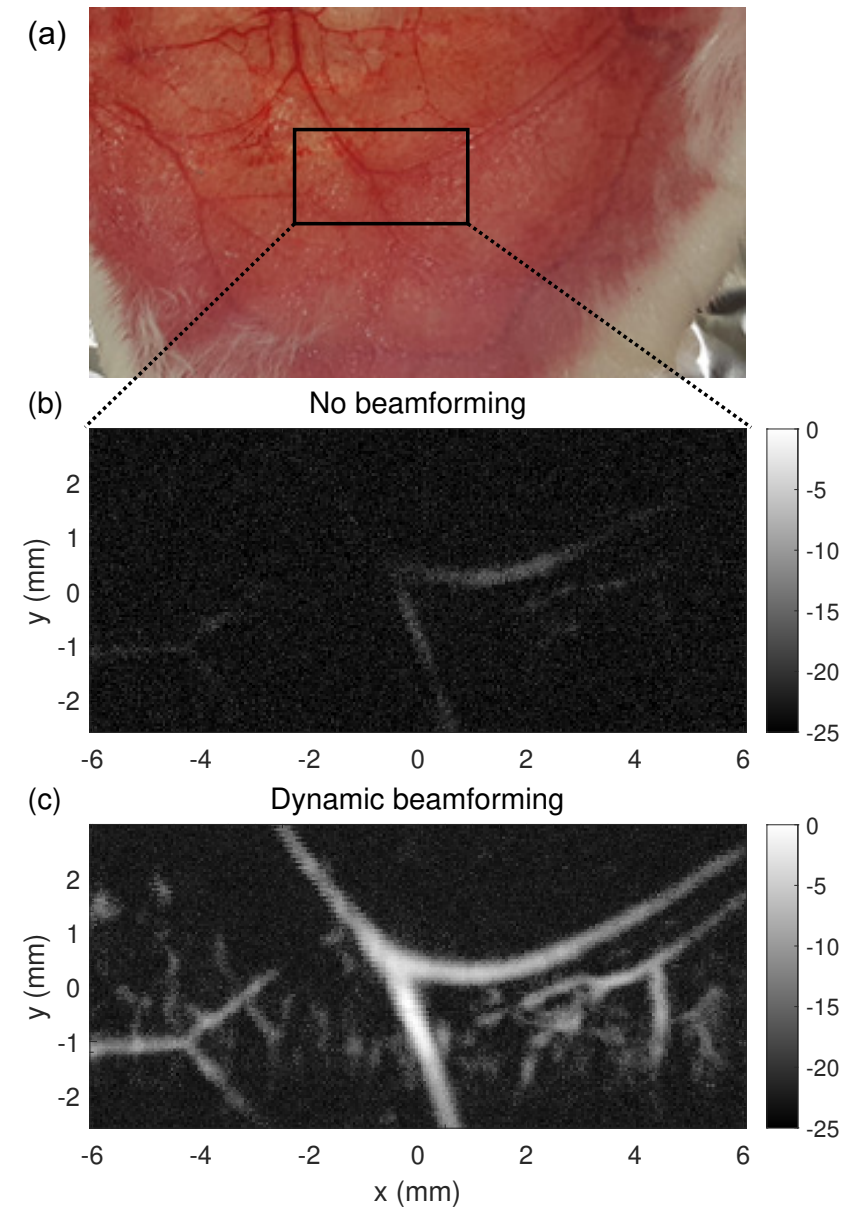

Fig. 3. Ex vivo rabbit ear 2D PAM imaging. (a) Photograph of the excised rabbit ear, where the black rectangle indicates the scanned area. PAM images of the rabbit ear vasculature comparing (b) no-beamforming and (c) dynamic beamforming.

Images of the vasculature of a rabbit's ear were performed in order to evaluate the system in a more realistic scenario. Fig. 3 displays a compared view of no-beamforming and dynamic beamforming approaches in an area of $1.2 \times 0.6 \mathrm{~cm}$, using step increments of $50 \mu \mathrm{m}$. Again, static beamforming post-processing show a noticeably better SNR and a sharper image, while the image resulting from the no-beamforming strategy is hardly visible.

\section{CONCLUSIONS}

We used a beamforming method in an array-based OR-PAM systems in order to evaluate its applicability for large-area scanning. A PLD controlled by a voltage driver module combined with an optic scheme of two plano-convex lenses is used as light source. The lateral resolution of the system is measured to be $200 \times 119 \mu \mathrm{m}^{2}$. Images of polyethylene tubes filled with India ink in tissue mimicking phantoms were acquired comparing three different beamforming strategies, demonstrating that the so-called dynamic beamforming achieves better contrast and SNR by adjusting the delayed signals following a DAS algorithm. Images of the microvasculature of a rabbit's ear to test its applicability in ex-vivo organs. Although a limitation of the system is the scanning time, this can be greatly improved by increasing the PRF of the system by using faster scanning techniques, such as MEMS or galvanometers scanners.

\section{ACKNOWLEDGMENT}

This research has been supported by the Spanish Ministry of Science, Innovation and Universities through grant "Juan de la Cierva - Incorporación” (IJC2018-037897-I), and program "Proyectos I+D+i 2019" (PID2019-111436RB-C22). Action co-financed by the European Union through the Programa Operativo del Fondo Europeo de Desarrollo Regional (FEDER) of the Comunitat Valenciana 2014-2020 (IDIFEDER/2018/022). A.C. received financial support from Generalitat Valenciana and Universitat Politècnica de València through the grants APOSTD/2018/229 and program PAID-10-19, respectively.

\section{REFERENCES}

[1] P. Beard, Biomedical photoacoustic imaging, Interface focus 1 (4) (2011) 602-631.

[2] L. V. Wang, J. Yao, A practical guide to photoacoustic tomography in the life sciences, Nature methods 13 (8) (2016) 627.

[3] T. J. Allen, B. Cox, P. C. Beard, Generating photoacoustic signals using high-peak power pulsed laser diodes, in: Photons Plus Ultrasound: Imaging and Sensing 2005: The Sixth Conference on Biomedical Thermoacoustics, Optoacoustics, and Acousto-optics, Vol. 5697, International Society for Optics and Photonics, 2005, pp. 233 242.

[4] M. Erfanzadeh, Q. Zhu, Photoacoustic imaging with lowcost sources; a review, Photoacoustics 14 (2019) 1-11.

[5] T. J. Allen, O. Ogunlade, E. Zhang, P. C. Beard, Large area laser scanning optical resolution photoacoustic microscopy using a fibre optic sensor, Biomedical optics express 9 (2) (2018) 650-660.

[6] R. Bitton, R. Zemp, J. Yen, L. V. Wang, K. K. Shung, A 3 -d high-frequency array based 16 channel photoacoustic microscopy system for in vivo micro-vascular imaging, IEEE transactions on medical imaging 28 (8) (2009) 1190-1197.

[7] L. Song, K. I. Maslov, K. K. Shung, L. V. Wang, Ultrasound-array-based real-time photoacoustic 
microscopy of human pulsatile dynamics in vivo, Journal of biomedical optics 15 (2) (2010) 021303.

[8] P. Hajireza, W. Shi, P. Shao, S. Kerr, R. Zemp, Opticalresolution photoacoustic micro-endoscopy using imageguide fibers and fiber laser technology, in: Photons Plus Ultrasound: Imaging and Sensing 2011, Vol. 7899, International Society for Optics and Photonics, 2011, p. $78990 \mathrm{P}$.

[9] A. Hariri, A. Fatima, N. Mohammadian, S. Mahmoodkalayeh, M. A. Ansari, N. Bely, M. R. Avanaki, Development of low-cost photoacoustic imaging systems using very low-energy pulsed laser diodes, Journal of biomedical optics 22 (7) (2017) 075001.

[10] B. Yin, D. Xing, Y. Wang, Y. Zeng, Y. Tan, Q. Chen, Fast photoacoustic imaging system based on 320-element linear transducer array, Physics in Medicine \& Biology 49 (7) (2004) 1339. 